\title{
Global unbounded solutions of the Fujita equation in the intermediate range*
}

\author{
Peter Poláčik ${ }^{\dagger}$ \\ School of Mathematics, University of Minnesota, \\ Minneapolis, MN 55455, USA \\ Eiji Yanagida \\ Department of Mathematics, Tokyo Institute of Technology, \\ Meguro-ku, Tokyo 152-8551, Japan
}

\begin{abstract}
We consider the Fujita equation $u_{t}=\Delta u+u^{p}$ on $\mathbb{R}^{N}$ with $N \geq 3$. We prove the existence of global, unbounded solutions for $p_{S}<p<$ $p_{J L}$, where $p_{S}:=(N+2) /(N-2)$ and

$$
p_{J L}:= \begin{cases}\frac{(N-2)^{2}-4 N+8 \sqrt{N-1}}{(N-2)(N-10)} & \text { if } N>10 \\ \infty & \text { if } N \leq 10\end{cases}
$$

Previously, it was known that global, unbounded solutions exist for $p \geq p_{J L}$, whereas for $p<p_{S}$ there are no such solutions.
\end{abstract}

Keywords: supercritical semilinear parabolic equation; unbounded global solutions, threshold solutions.

*This research was carried out during a visit of P. Poláčik to the Tokyo Institute of Technology.

†Supported in part by NSF Grant DMS-1161923.

${ }^{\ddagger}$ Supported in part by JSPS Grant-in-Aid for Scientific Research (A) (No. 24244012). 


\section{Introduction}

We consider the Cauchy problem

$$
\begin{array}{ll}
u_{t}=\Delta u+u^{p}, & x \in \mathbb{R}^{N}, t>0, \\
u(x, 0)=u_{0}(x), & x \in \mathbb{R}^{N},
\end{array}
$$

where $p>1$, and $u_{0} \in C\left(\mathbb{R}^{N}\right) \cap L^{\infty}\left(\mathbb{R}^{N}\right)$ is a nonnegative function. With an exception of a few introductory remarks, we shall also assume that $u_{0}$ is radially symmetric, that is, $u_{0}(x)$ depends on $|x|$ only.

Problem (1.1), (1.2) and its counterpart on bounded domains have been widely studied as model problems in the theory of blowup and related topics. Equation (1.1) also appears as a scaling limit of more general superlinear equations whose nonlinearities have polynomial growth. As such, (1.1) plays an important role in the theory of semilinear parabolic equations. For a general background in blowup for parabolic equations and the role of equation (1.1) the reader can consult the monographs [15, 27], for example.

Problem (1.1), (1.2) has a unique (classical) solution $u$ defined on some time interval $[0, T)$ with $0<T \leq \infty$. We take $T=T\left(u_{0}\right)$ maximal possible, so the solution cannot be extended beyond $T$. If $T<\infty$, then necessarily the solution blows up at $T:\|u(\cdot, t)\|_{L^{\infty}\left(\mathbb{R}^{N}\right)} \rightarrow \infty$ as $t \nearrow T$. If $T=\infty$, we say that the solution is global. In this case, two possibilities can occur, in principle: either $u$ is bounded or

$$
\limsup _{t \rightarrow \infty}\|u(\cdot, t)\|_{L^{\infty}\left(\mathbb{R}^{N}\right)}=\infty .
$$

The latter is sometimes referred to as infinite time blowup or growup of $u$. One of the fundamental problems concerning equation (1.1) is whether the infinite time blowup can actually occur for some $u_{0}$ or not.

To discuss this problem in more specific terms, first recall that blowup solutions of (1.1) exist for all $p>1$ : $u_{0}$ identical to a positive constant gives the simplest example. For $1<p \leq p_{F}, p_{F}:=1+2 / N$ being the Fujita exponent, it is even true that all positive solutions blow up (see [10, 15, 17, 27]). If $p>p_{F}$, there exist positive bounded solutions. Examples are provided by forward self-similar solutions (see [14]) and, for $p \geq p_{S}$, also by positive steady states. Here $p_{S}$ is the Sobolev critical exponent:

$$
p_{S}:= \begin{cases}\frac{N+2}{N-2} & \text { if } N>2 \\ \infty & \text { if } N \leq 2 .\end{cases}
$$


The problem of existence of global, unbounded solutions is more delicate. In the subcritical range, $p<p_{S}$, not only are all global positive solutions bounded, they even decay to zero as $t \rightarrow \infty[23,24,27]$ (this result uses the radial symmetry of $u_{0}$; without the radial symmetry it has only been proved for a smaller range of exponents [3, 24, 27]). For the Sobolev critical exponent, $p=p_{S}$, formal asymptotic expansions suggest that global unbounded solutions exist for $N=3,4$ (see [7]), but there does not seem to be a rigorous proof of the existence or nonexistence.

As for the supercritical case, the problem has been resolved for $p \geq p_{J L}$, where $p_{J L}$ is the Joseph-Lundgren exponent:

$$
p_{J L}:= \begin{cases}\frac{(N-2)^{2}-4 N+8 \sqrt{N-1}}{(N-2)(N-10)} & \text { if } N>10 \\ \infty & \text { if } N \leq 10\end{cases}
$$

Positive global solutions with

$$
\liminf _{t \rightarrow \infty}\|u(\cdot, t)\|_{L^{\infty}\left(\mathbb{R}^{N}\right)}<\limsup _{t \rightarrow \infty}\|u(\cdot, t)\|_{L^{\infty}\left(\mathbb{R}^{N}\right)}=\infty
$$

as well as solutions with

$$
\lim _{t \rightarrow \infty}\|u(\cdot, t)\|_{L^{\infty}\left(\mathbb{R}^{N}\right)}=\infty
$$

have been found by the present authors in [25]. The latter case was studied in more detail in $[8,9,22]$, where various rates of growup were found.

In the intermediate range, $p_{S}<p<p_{J L}$, the question whether the solution of (1.1), (1.2) can be global and unbounded has been open until now, although it has been answered in negative under additional assumptions on $u_{0}$. In particular, Mizoguchi [20] proved that the solution cannot be global and unbounded, provided that $u_{0}$ is a $C^{1}$ nonnegative radially symmetric function with compact support and, viewed as a function of $r=|x| \in[0, \infty)$, $u_{0}$ has only finitely many local minima. Matano and Merle $[18,19]$ proved this result under weaker conditions on $u_{0}$ (still assuming the radial symmetry). Namely, it is sufficient that $u_{0} \in H^{1}\left(\mathbb{R}^{N}\right)$ or that $u_{0}$, viewed again as a function of $r$, has only finitely many intersections with the singular steady state $\varphi_{\infty}$ (see (2.1) below for the definition of $\varphi_{\infty}$ ).

In this paper, we prove that without any such additional conditions on $u_{0}$, the solution of $(1.1),(1.2)$ can actually be global and unbounded. The following is our main theorem. 
Theorem 1.1. Let $p_{S}<p<p_{J L}$. There exists a radially symmetric and radially nonincreasing nonnegative function $u_{0} \in C\left(\mathbb{R}^{N}\right)$ such that the solution $u$ of (1.1), (1.2) has the following properties: $u$ is global,

$$
0<u(x, t) \leq C|x|^{-\frac{2}{p-1}} \quad\left(x \in \mathbb{R}^{N} \backslash\{0\}, t>0\right),
$$

where $C$ is a constant, and

$$
\limsup _{t \rightarrow \infty}\|u(\cdot, t)\|_{L^{\infty}\left(\mathbb{R}^{N}\right)}=\infty .
$$

Condition (1.5) in particular implies that the solution $u$ is localized: $u(x, t) \rightarrow 0$ as $|x| \rightarrow \infty$ uniformly in $t$.

We remark that our existence result for the intermediate range is not as complete as for the range $p \geq p_{J L}$, cp. [25]. Namely, it does not assert that both possibilities (1.3) and (1.4) can occur. While we do show the existence of solutions satisfying (1.3) (see relations (3.3) at the end of the proof of Theorem 1.1), it is not clear if our method can be modified so as to yield solutions satisfying (1.4).

There is some flexibility in choosing the initial value $u_{0}$ in Theorem 1.1. As one can easily check (specifically, see condition (3.2) in the proof of Theorem 1.1), our construction shows that there is a nonempty open set $G$ in a suitable weighted space $L^{\infty}\left(\mathbb{R}^{N}, \rho\right)$ such that Theorem 1.1 holds for each radially symmetric and radially nonincreasing nonnegative function $u_{0} \in G$. In particular, $u_{0}$ can be chosen smooth.

For completeness of the discussion, let us briefly mention related results for the Dirichlet problem

$$
\begin{array}{ll}
u_{t}=\Delta u+u^{p}, & x \in \Omega, t>0, \\
u(x, t)=0, & x \in \partial \Omega, t>0, \\
u(x, 0)=u_{0}(x), & x \in \mathbb{R}^{N} .
\end{array}
$$

If $\Omega$ is a ball in $\mathbb{R}^{N}$ centered at the origin and $u_{0} \in C(\bar{\Omega})$ is a radially symmetric nonnegative function, then for all $p>1, p \neq p_{S}$, the solution of (1.6)-(1.8) has to be bounded if it is global; see $[5,11,21]$ for the proofs in the supercritical case $p>p_{S}$, and $[4,6,12,26]$ in the subcritical case $p<p_{S}$ (in the subcritical case, the result is valid without the radial symmetry requirement and for any smooth bounded domain). The proofs of these results, their discussion, and additional references can also be found in [27]. On the 
other hand, if $N \geq 3$ and $p=p_{S}$, then for a suitable radially symmetric $u_{0}$, the solution of (1.6)-(1.8) is global and unbounded, see [11] or [27].

The rest of this paper is organized as follows. In the next section, we collect preliminary results. We discuss in some detail threshold solutions of (1.1), which play a crucial role in our construction. Theorem 1.1 is proved in Section 3.

In the remainder of this paper, we shall only consider initial values $u_{0}$ in $X_{+}$, where

$$
X_{+}:=\left\{u_{0} \in C\left(\mathbb{R}^{N}\right) \cap L^{\infty}\left(\mathbb{R}^{N}\right): u_{0} \geq 0 \text { and } u_{0} \text { is radially symmetric }\right\} .
$$

At some occasions, when there is no danger of confusion, we abuse the notation slightly and view a radial function $u_{0}$ of $x \in \mathbb{R}^{N}$ as a function of $r=|x| \in[0, \infty)$. The same applies to the solution of (1.1), (1.2). Note that, by uniqueness, the solution is radially symmetric in $x$. Also, when $u_{0}$ is radially decreasing, then so is the solution for each $t$. This follows from the maximum principle applied to the function $u_{x_{1}}$ on $\left\{x \in \mathbb{R}^{N}: x_{1} \geq 0\right\}$.

Below we frequently use the notation

$$
B_{R}:=\left\{x \in \mathbb{R}^{N}:|x|<R\right\} .
$$

In the rest of the paper we assume that

$$
p_{S}<p<p_{J L}
$$

\section{Properties of threshold solutions}

First we recall some known results concerning the structure of the radial steady states of (1.1).

Let

$$
\varphi_{\infty}(x):=L|x|^{-\frac{2}{p-1}} \quad\left(x \in \mathbb{R}^{N} \backslash\{0\}\right)
$$

with

$$
L:=\left\{\frac{2}{p-1}\left(N-2-\frac{2}{p-1}\right)\right\}^{\frac{1}{p-1}} .
$$

This is a singular radial steady state of (1.1). There is a one-parameter family of classical radial positive steady states $\varphi_{\alpha}$, parameterized by $\alpha=\varphi_{\alpha}(0)$. In view of the scaling invariance of (1.1), one has

$$
\varphi_{\alpha}(x)=\alpha \varphi_{1}\left(\alpha^{\frac{p-1}{2}} x\right) \quad(\alpha>0) .
$$


The graphs of any two radial steady states $\varphi_{\alpha}, \varphi_{\beta}, 0<\alpha<\beta \leq \infty$, intersect each other infinitely many times $[28,13]$. Moreover, the radial steady states are unstable. In particular, the following is true.

Lemma 2.1. If $u_{0} \in X_{+}$and $u_{0} \leq \varphi_{\alpha}, u_{0} \not \equiv \varphi_{\alpha}$ for some $\alpha \in(0, \infty]$, then the solution of (1.1), (1.2) satisfies

$$
\lim _{t \rightarrow \infty}\|u(\cdot, t)\|_{L^{\infty}\left(\mathbb{R}^{N}\right)}=0 .
$$

For $\alpha<\infty$, this is a part of the instability result of [13]; the case $\alpha=\infty$ is treated in [11].

We next examine threshold solutions of (1.1). Let $u_{0} \in X_{+} \backslash\{0\}$. We say that $u_{0}$ is a threshold initial value and the solution of (1.1), (1.2) is a threshold solution if for each $\tilde{u}_{0} \in X_{+} \backslash\left\{u_{0}\right\}$ the following statements are valid:

(t1) if $\tilde{u}_{0} \leq u_{0}$, then the solution of $(1.1)$ with $u(\cdot, 0)=\tilde{u}_{0}$ is global and satisfies (2.2);

(t2) if $\tilde{u}_{0} \geq u_{0}$, then the solution of $(1.1)$ with $u(\cdot, 0)=\tilde{u}_{0}$ blows up.

In literature, threshold solutions are usually defined a little differently, requiring merely that (t1), (t2) hold for functions $\tilde{u}_{0}$ of the form $\tilde{u}_{0}=\lambda u_{0}$, $\lambda>0$. The next lemma implies that in all cases we deal with, these definitions are equivalent. Also, we will need the fact that the threshold solutions blow up.

Lemma 2.2. Let $u_{0} \in X_{+} \cap H^{1}\left(\mathbb{R}^{N}\right)$. Suppose that there are sequences $\left\{\underline{u}_{n}\right\}$, $\left\{\bar{u}_{n}\right\}$ in $X_{+}$such that $\underline{u}_{n} \rightarrow u_{0}, \bar{u}_{n} \rightarrow u_{0}$, as $n \rightarrow \infty$, uniformly on $\mathbb{R}^{N}$, and the following conditions are satisfied for all $n=1,2, \ldots$ :

(p1) $\underline{u}_{n+1} \geq \underline{u}_{n}, \underline{u}_{n+1} \not \equiv \underline{u}_{n}$;

(p2) $\bar{u}_{n} \geq \bar{u}_{n+1}, \bar{u}_{n+1} \not \equiv \bar{u}_{n}$;

(p3) the solution of $(1.1)$ with $u(\cdot, 0)=\underline{u}_{n}$ is global;

(p4) the solution of $(1.1)$ with $u(\cdot, 0)=\bar{u}_{n}$ blows up.

Then $u_{0}$ is a threshold initial value and the solution of (1.1) (1.2) blows up. 
Remark 2.3. Since $u_{0} \in X_{+} \cap H^{1}\left(\mathbb{R}^{N}\right)$, it can be assumed without loss of generality that the functions $\underline{\underline{u}}_{n}, n=1,2, \ldots$, have compact supports. Indeed, being a radial $H^{1}$-function, $u_{0}$ decays to 0 as $|x| \rightarrow \infty[2$, Lemma A.II]. So there is a sequence $\left\{\phi_{n}\right\} \subset X_{+}$of functions with compact supports such that $\phi_{n} \nearrow u_{0}$ in $L^{\infty}\left(\mathbb{R}^{N}\right)$. If the original $\underline{\mathrm{u}}_{n}$ do not have compact supports, we can simply replace them with the function $\min \left\{\phi_{n}, \underline{u}_{n}\right\}$. Note that (p3) remains valid for the new functions by the comparison principle.

There are several ways to prove Lemma 2.2. We shall derive it from results of [18], [19], and [16]. Under slightly different assumptions, arguments of [20] could also be used.

Proof of Lemma 2.2. The fact that, under the given assumptions, the solution of (1.1), (1.2) blows up is proved in [19, Proof of Theorem 5.1]. Specifically, in the arguments following formula (5.25) in [19], it makes no difference if in place of $\lambda^{*} v$ and $\lambda_{n} v$ one considers $u_{0}$ and $\bar{u}_{n}$, respectively. By comparison, it now follows that statement (t2) holds for each $\tilde{u}_{0} \in X_{+} \backslash\left\{u_{0}\right\}$. It remains to prove that $(\mathrm{t} 1)$ holds.

Suppose, for a contradiction, that for some $\tilde{u}_{0} \in X_{+} \backslash\left\{u_{0}\right\}$ with $\tilde{u}_{0} \leq u_{0}$ the solution $\tilde{u}$ of $(1.1)$ with $u(\cdot, 0)=\tilde{u}_{0}$ blows up at some $\tilde{T}$. We intend to apply [16, Theorem 2] to conclude that the proper extension of the solution $u$ blows up completely at some time $T_{c}<\tilde{T}$. Let us first recall the terminology (see, for example, $[1,11,19,27]$ for more detailed discussions of these concepts). The proper extension $U$ of $u$ is defined by

$$
U(x, t)=\lim _{k \rightarrow \infty} v_{k}(x, t) \quad\left((x, t) \in \mathbb{R}^{N} \times[0, \infty)\right),
$$

where $v_{k}$ is the solution of the problem

$$
\begin{array}{ll}
v_{t}=\Delta v+f_{k}(v), & x \in \mathbb{R}^{N}, t>0, \\
v(x, 0)=u_{0}(x), & x \in \mathbb{R}^{N},
\end{array}
$$

with $f_{k}(v):=\max \left\{v^{p}, k\right\}$. Since the $f_{k}$ are globally Lipschitz and $f_{k+1} \geq f_{k}$, the solutions $v_{k}$ are global and $v_{k+1} \geq v_{k}$. Consequently, the limit in (2.3), finite or infinite, exists for all $(x, t) \in \mathbb{R}^{N} \times[0, \infty)$. Set

$$
T_{c}:=\sup \left\{t_{0}>0: U\left(x, t_{0}\right)<\infty \text { for a.e. } x \in \mathbb{R}^{N}\right\} .
$$

If $T_{c}<0$, we say that the solution $u$ (or, more precisely, its proper extension $U$ ) blows up completely at $T_{c}$. 
Theorem 2 of [16] deals with solutions whose initial conditions are related as in our case: $\tilde{u}_{0} \leq u_{0}$ and $\tilde{u}_{0} \not \equiv u_{0}$. Its conclusion is that $T_{c}<\tilde{T}$. Although [16] apparently focuses on problems on bounded domains, as noted in [19, Lemma 5.7], [16, Theorem 2] applies in our situation if we can guarantee that for some $R>0$

$$
\sup _{0<t<\tilde{T},|x| \geq R} \tilde{u}(x, t)<\infty .
$$

To verify this condition, we estimate the function $U$ and then use the fact that the comparison principle holds for the proper extension of solutions of (1.1) (see [19, Proposition 5.9]). In particular, for each $t \in[0, \tilde{T})$ one has

$$
\tilde{u}(x, t) \leq U(x, t) \text { for a.e. } x \in \mathbb{R}^{N} .
$$

Estimating $U$, we will at the same time get a contradiction to the conclusion $T_{c}<\tilde{T}$.

In the sequel, we assume without loss of generality that the functions $\underline{\mathrm{u}}_{n}$, $n=1,2, \ldots$, have compact supports (cp. Remark 2.3).

Let $U_{n}$ be the solution of $(1.1)$ with $U_{n}(\cdot, 0)=\underline{\mathrm{u}}_{n}$. By (p3), this solution is global and, by (p1) and the comparison principle, $U_{n+1} \geq U_{n}$. Consequently, as $n \rightarrow \infty$, the limit of $U_{n}(x, t)$, possibly infinite, exists for all $(x, t) \in$ $\mathbb{R}^{N} \times[0, \infty)$. Proposition 5.5 of [19] says that this limit coincides with the proper extension $U$ of $u$ :

$$
\lim _{n \rightarrow \infty} U_{n}(x, t)=U(x, t) .
$$

As $\underline{\mathrm{u}}_{n} \rightarrow u_{0}$ in $L^{\infty}\left(\mathbb{R}^{N}\right)$, the functions $U_{n}(x, 0)=\underline{\mathrm{u}}_{n}, n=1,2, \ldots$, are uniformly bounded. In this situation, [18, Corollary 3.3] shows that for any $T^{*}>0$ and $\delta>0$ there is a constant $C=C\left(T^{*}, \delta\right)$ such that

$$
U(x, t) \leq C\left(1+|x|^{-\frac{2}{p-1}}\right) \quad\left(x \in \mathbb{R}^{N} \backslash\{0\}, \delta \leq t \leq T^{*}-\delta\right) .
$$

We now return to (2.4). Take $\delta=\tilde{T} / 2, T^{*}=\tilde{T}+\delta$. Since $\tilde{u}$ is uniformly bounded on $\mathbb{R}^{N} \times[0, \delta]$, we see that (2.4) holds for any $R>0$ due to the relations (2.5) and (2.6). Thus the application of [16, Theorem 2] is legitimate and we have $T_{c}<\tilde{T}<\infty$. On the other hand, (2.6) clearly implies that $T_{c}=\infty$, a contradiction.

This contradiction shows that for each $\tilde{u}_{0} \in X_{+} \backslash\left\{u_{0}\right\}, \tilde{u}_{0} \leq u_{0}$ the solution $\tilde{u}$ of $(1.1)$ with $u(\cdot, 0)=\tilde{u}_{0}$ must be global. To show that $u_{0}$ is a threshold initial value, it remains to be verified that

$$
\|\tilde{u}(\cdot, t)\|_{L^{\infty}\left(\mathbb{R}^{N}\right)} \rightarrow 0 \text { as } t \rightarrow \infty .
$$


We can argue by comparison again. By [19, Theorem 5.14], the extension $U(\cdot, t)$ is a classical solution of (1.1) for all sufficiently large $t$ and

$$
\|U(\cdot, t)\|_{L^{\infty}\left(\mathbb{R}^{N}\right)} \rightarrow 0 \text { as } t \rightarrow \infty .
$$

Using this and relation (2.5), which holds for each $0<t<\tilde{T}=\infty$, we obtain (2.7).

The following result will help us find threshold solutions in ordered families of initial data.

Corollary 2.4. Let $0 \leq a<b \leq \infty$ and let $u_{0}^{\lambda}, \lambda \in[a, b)$, be a family of functions in $X_{+} \cap H^{1}\left(\mathbb{R}^{N}\right) \backslash\{0\}$ such that $u_{0}^{\lambda} \leq u_{0}^{\mu}$, $u_{0}^{\lambda} \not \equiv u_{0}^{\mu}$, whenever $\lambda<\mu$, and the map $\lambda \mapsto u_{0}^{\lambda}:[a, b) \rightarrow L^{\infty}\left(\mathbb{R}^{N}\right)$ is continuous. Let $u^{\lambda}$ denote the solution of (1.1), (1.2) with $u_{0}=u_{0}^{\lambda}$. Then one of the following statements is valid:

(s1) For each $\lambda \in[a, b)$ the solution $u^{\lambda}$ is global.

(s2) For each $\lambda \in[a, b)$ the solution $u^{\lambda}$ blows up.

(s3) There is a unique $\lambda_{0} \in(a, b)$ such that $u^{\lambda_{0}}$ is a threshold solution.

Proof. Assume that (s1), (s2) do not hold. We prove that (s3) holds for

$$
\lambda_{0}:=\sup \left\{\mu \in[a, b): u^{\lambda} \text { is global for each } a \leq \lambda \leq \mu\right\} .
$$

Since (s1), (s2) do not hold, $a \leq \lambda_{0}<b$. If $\lambda_{0}>a$, then $u^{\lambda_{0}}$ is a threshold solution by a direct application of Lemma 2.2.

We next rule out the case $\lambda_{0}=a$. Assuming it holds, the solution $u^{\lambda_{0}}$ is necessarily global and then, by comparison, so is each solution with initial value $u_{0} \in X_{+}, u_{0} \leq u_{0}^{\lambda_{0}}$. By assumptions and the definition of $\lambda_{0}$, Lemma 2.2 again applies to $u_{0}^{\lambda_{0}}$. However, this time it gives a contradiction to the fact that the solution $u^{\lambda_{0}}$ is global.

To guarantee that some solutions in a family blow up, we shall usually rely on the following sufficient condition (see for example [15, 17, 27]).

Lemma 2.5. Let $u_{0} \in X_{+} \cap H^{1}\left(\mathbb{R}^{N}\right)$. If

$$
E\left(u_{0}\right):=\int_{\mathbb{R}^{N}}\left(\frac{\left|\nabla u_{0}(x)\right|^{2}}{2}-\frac{u_{0}^{p+1}(x)}{p+1}\right) d x<0,
$$

then the solution of (1.1), (1.2) blows up. 
The next lemma gives an a priori bound on global solutions. A similar estimate under different assumptions (and with a different proof) is given in [20, Lemma 2.2].

Lemma 2.6. There is a constant $C=C(p, N)$ with the following property: If $u_{0} \in X_{+}$is radially nonincreasing and the solution $u(x, t)$ of $(1.1),(1.2)$ is global, then

$$
u(x, t) \leq C|x|^{-\frac{2}{p-1}} \quad\left(x \in \mathbb{R}^{N} \backslash\{0\}, t \geq 0\right) .
$$

Proof. Recall that the solution $u(\cdot, t)$ is radially symmetric and radially nonincreasing for each $t$.

Choose a smooth function $\psi \in X_{+}$with support in $B_{2}$ and with $\psi(0)=$ $\|\psi\|_{L^{\infty}\left(\mathbb{R}^{N}\right)}=1$. Using Lemma 2.5 , one shows easily that if $d=d(p, N)$ is sufficiently large, then the solution $\bar{u}$ of $(1.1)$ with $\bar{u}(\cdot, 0)=d \psi$ blows up. Therefore the global solution $u$ cannot be above $d \psi$ at any time. In other words, for each $t_{0}$ there is at least one $x \in B_{2}$ such that $u\left(x, t_{0}\right) \leq d \psi(x) \leq d$. Consequently, in view of the radial nonincrease of $u, u\left(x_{0}, t_{0}\right) \leq d$ if $\left|x_{0}\right|=2$. The same applies to the function $\lambda^{2 /(p-1)} u\left(\lambda x, \lambda^{2} t\right)$, which is also a global solution of (1.1) for any $\lambda>0$. Hence we obtain

$$
\lambda^{2 /(p-1)} u\left(\lambda x_{0}, \lambda^{2} t_{0}\right) \leq d \quad\left(\left|x_{0}\right|=2, t_{0} \geq 0, \lambda>0\right) .
$$

Given any $x \in \mathbb{R}^{N} \backslash\{0\}$ and $t \geq 0$, take $\lambda=|x| / 2, t_{0}=\lambda^{-2} t, x_{0}=2 x /|x|$, to obtain (2.8) with $C:=d 2^{2 /(p-1)}$.

\section{Proof of Theorem 1.1}

Throughout this section $u\left(\cdot, t, u_{0}\right)$ stands for the (maximally defined) radially symmetric solution of (1.1), (1.2).

We shall construct a sequence $\left\{\left(u_{k}, R_{k}, t_{k}, \bar{t}_{k}, \delta_{k}\right)\right\}$ in $X_{+} \times(0, \infty)^{4}$ such that the following statements hold true for each $k=1,2, \ldots$.

(i) $\bar{t}_{k}>t_{k}+1$.

(ii) $u_{k}$ is radially nonincreasing, $u_{k}>0$ on $\bar{B}_{R_{k}}$, and $u_{k} \equiv 0$ on $\mathbb{R}^{N} \backslash B_{R_{k}+1}$.

(iii) The solution $u\left(x, t, u_{k}\right)$ is global and

$$
u\left(0, t_{k}, u_{k}\right)>k, \quad \lim _{t \rightarrow \infty}\left\|u\left(\cdot, t, u_{k}\right)\right\|_{L^{\infty}\left(\mathbb{R}^{N}\right)}=0 .
$$


(iv) For any $u_{0} \in C\left(\mathbb{R}^{N}\right)$ with $\left\|u_{k}-u_{0}\right\|_{L^{\infty}\left(\mathbb{R}^{N}\right)}<\delta_{k}$, the solution $u\left(\cdot, t, u_{0}\right)$ is defined for $t \in\left[0, \bar{t}_{k}\right]$ and satisfies

$$
\left\|u\left(\cdot, t_{k}, u_{0}\right)\right\|_{L^{\infty}\left(\mathbb{R}^{N}\right)}>k, \quad\left\|u\left(\cdot, \bar{t}_{k}, u_{0}\right)\right\|_{L^{\infty}\left(\mathbb{R}^{N}\right)}<\frac{1}{k} .
$$

(v) $R_{k+1} \geq R_{k}+1, t_{k+1}>\bar{t}_{k}, \delta_{k+1}<\delta_{k} / 2$.

(vi) $u_{k+1} \equiv u_{k}$ on $B_{R_{k}}$ and $u_{k} \leq u_{k+1} \leq u_{k}+\delta_{k} / 2$ on $\mathbb{R}^{N} \backslash B_{R_{k}}$.

Suppose for a while that such a sequence $\left\{\left(u_{k}, R_{k}, t_{k}, \bar{t}_{k}, \delta_{k}\right)\right\}$ has been found. We now use it to prove Theorem 1.1.

Proof of Theorem 1.1. Take any radially nonincreasing function $u_{0} \in X_{+}$ with

$$
\left\|u_{k}-u_{0}\right\|_{L^{\infty}\left(\mathbb{R}^{N}\right)}<\delta_{k} \quad(k=1,2, \ldots) .
$$

For example, we can define $u_{0}$ by

$$
u_{0}(x) \equiv u_{k}(x) \quad\left(x \in B_{R_{k}}, k=1,2, \ldots\right),
$$

which is legitimate by (vi). By (ii), $u_{0} \in X_{+}$, it is radially nonincreasing, and relations (v) and (vi) imply that it satisfies (3.2).

By (3.2), statement (v) applies to $u_{0}$ for each $k$. As $t_{k} \rightarrow \infty$, by (i) and $(\mathrm{v})$, we see that the solution $u\left(\cdot, t, u_{0}\right)$ is global and $(3.1)$ holds for $k=$ $1,2, \ldots$ Thus

$$
\liminf _{t \rightarrow \infty}\|u(\cdot, t)\|_{L^{\infty}\left(\mathbb{R}^{N}\right)}=0, \quad \limsup _{t \rightarrow \infty}\|u(\cdot, t)\|_{L^{\infty}\left(\mathbb{R}^{N}\right)}=\infty .
$$

Finally, Lemma 2.6 yields the bound (1.5) on the solution $u(x, t)=u\left(x, t, u_{0}\right)$. The proof of Theorem 1.1 is complete.

We now carry out a construction of the sequence $\left\{\left(u_{k}, R_{k}, t_{k}, \bar{t}_{k}, \delta_{k}\right)\right\}$. Since we only deal with radial functions, in this construction we view them as functions of $r=|x| \in[0, \infty)$. We proceed by induction.

STEP 1. Choose a constant $\epsilon>0$ such that $\epsilon<\varphi_{\infty}$ on $[0,2]$. For each $R>0$, we define a piecewise linear, nonincreasing function $u_{1}^{R}(r)$ by

$$
u_{1}^{R}(r)= \begin{cases}\epsilon & (r \in[0, R]), \\ \epsilon-\epsilon(r-R) & (r \in[R, R+1]), \\ 0 & (r \geq R+1) .\end{cases}
$$


Obviously, for $R=1$ we have $u_{1}^{R}<\varphi_{\infty}$, hence

$$
\lim _{t \rightarrow \infty}\left\|u\left(\cdot, t, u_{1}^{R}\right)\right\|_{L^{\infty}\left(\mathbb{R}^{N}\right)}=0
$$

by Lemma 2.1. On the other hand, Lemma 2.5 implies that for $R$ sufficiently large, the solution $u\left(\cdot, \cdot, u_{1}^{R}\right)$ blows up. Hence, by Corollary 2.4 , there is a unique $R^{*} \in(1, \infty)$ such that $u_{1}^{R^{*}}$ is a threshold initial value. Let $t_{1}>0$ be the blowup time of $u\left(\cdot, \cdot, u_{1}^{R^{*}}\right)$ (the threshold solution does blow up by Lemma 2.2). Observe that if $R<R^{*}$ is sufficiently close to $R^{*}$, then the solution $u\left(\cdot, t, u_{1}^{R}\right)$ is global and satisfies

$$
u\left(0, t_{1}, u_{1}^{R}\right)=\left\|u\left(\cdot, t, u_{1}^{R}\right)\right\|_{L^{\infty}\left(\mathbb{R}^{N}\right)}>1, \quad \lim _{t \rightarrow \infty}\left\|u\left(\cdot, t, u_{1}^{R}\right)\right\|_{L^{\infty}\left(\mathbb{R}^{N}\right)}=0 .
$$

Indeed, the second condition in (3.4) is immediate from the definition of a threshold solution and the first one follows from the continuous dependence of the solutions on their initial data. Choose $R<R^{*}$ such that (3.4) holds and set $R_{1}:=R, u_{1}:=u_{1}^{R}$. With this choice, (ii), (iii) are met for $k=1$. By (3.4), there is $\bar{t}_{1}>t_{1}+1$ such that $u\left(0, \bar{t}_{1}, u_{1}\right)<1$. Using the continuity with respect to initial conditions, we find $\delta_{1}>0$ such that (iv) holds for $k=1$. This completes STEP 1.

STEP 2. Suppose that for some $n \geq 1,\left(u_{k}, R_{k}, t_{k}, \bar{t}_{k}, \delta_{k}\right) \in X_{+} \times(0, \infty)^{4}$, $k=1, \ldots, n$, have been constructed in such a way that the functions $u_{k}$, $k=1, \ldots, n$, are piecewise linear, (i)-(iv) hold for all $k=1, \ldots, n$, and (v), (vi) hold for $k<n$.

Let $0 \leq \epsilon<\min \left\{\delta_{n} / 2, u_{n}\left(R_{n}\right)\right\}$. For each $R \geq R_{n}+1$, we define a function $u_{n+1}^{R}(r)$ by

$$
u_{n+1}^{R}(r)= \begin{cases}\max \left\{\epsilon, u_{k}(r)\right\} & (r \in[0, R]), \\ \epsilon-\epsilon(r-R) & (r \in[R, R+1]), \\ 0 & (r \in[R+1, \infty]) .\end{cases}
$$

Since $u_{n} \geq u_{n}\left(R_{n}\right)>\epsilon$ on $\left[0, R_{n}\right]$ and $u_{n} \equiv 0$ on $\left[R_{n}+1, \infty\right), u_{n+1}^{R}$ is a (continuous) nonincreasing piecewise linear function satisfying $u_{n+1}^{R} \equiv u_{n}$ on $\left[0, R_{n}\right]$ and $u_{n} \leq u_{n+1}^{R} \leq u_{n}+\epsilon \leq u_{n}+\delta_{n} / 2$ on $\left[R_{n}, \infty\right)$. Moreover, if $\epsilon=0$, then $u_{n+1}^{R} \equiv u_{n}$ everywhere and for any $R$.

Let us first take $R=R_{n}+1$. Viewing $\epsilon$ as a parameter, we apply Corollary 2.4 to the family $u_{n+1}^{R}, \epsilon \in\left[0, \min \left\{\delta_{k} / 2, u_{k}\left(R_{k}\right)\right\}\right)$. Since for $\epsilon=0$ the initial 
value $u_{n+1}^{R} \equiv u_{n}$ gives a global solution, statement (s1) or statement (s3) of Corollary 2.4 holds. In either case, if $\epsilon>0$ is sufficiently small, then

$$
\lim _{t \rightarrow \infty}\left\|u\left(\cdot, t, u_{n+1}^{R}\right)\right\|_{L^{\infty}\left(\mathbb{R}^{N}\right)}=0 .
$$

Fix some $\epsilon \in\left(0, \min \left\{\delta_{k} / 2, u_{k}\left(R_{k}\right)\right\}\right)$ with this property.

Having fixed $\epsilon$, consider the family $u_{n+1}^{R}, R \geq R_{n}+1$. By Lemma 2.5, for $R$ sufficiently large, the solution $u\left(\cdot, t, u_{n+1}^{R}\right)$ blows up. Using this and (3.5) (with $R=R_{n}+1$ ), and applying Corollary 2.4 again, we find $R^{*}>R_{n}+1$ such that $u_{n+1}^{R^{*}}$ is a threshold initial value. As in STEP 1, we next choose $R<R^{*}$, sufficiently close to $R^{*}$, so that the solution $u\left(\cdot, t, u_{n+1}^{R}\right)$ is global, and

$$
\begin{aligned}
& u\left(0, t_{n+1}, u_{n+1}^{R}\right)=\left\|u\left(\cdot, t_{n+1}, u_{n+1}^{R}\right)\right\|>n+1, \\
& \lim _{t \rightarrow \infty}\left\|u\left(\cdot, t, u_{n+1}^{R}\right)\right\|_{L^{\infty}\left(\mathbb{R}^{N}\right)}=0,
\end{aligned}
$$

where $t_{n+1}>0$ is the blow up time of the threshold solution $u\left(\cdot, \cdot, u_{n+1}^{R^{*}}\right)$. Obviously, $t_{n+1}>\bar{t}_{n}$, as statement (iv) with $k=n$ applies to $u_{0}=u_{n+1}^{R^{*}}$. Setting $R_{n+1}:=R, u_{n+1}:=u_{n+1}^{R}$, we see that (ii), (iii) hold for $k=n+1$. By (3.6), there is $\bar{t}_{n+1}>t_{n+1}+1$ such that

$$
u\left(0, \bar{t}_{n+1}, u_{n+1}\right)<\frac{1}{n+1} .
$$

Using the continuity with respect to initial conditions, we find $\delta_{n+1} \in\left(0, \delta_{n} / 2\right)$ such that (iv) holds for $k=n+1$. By construction, relations (v), (vi) hold for $k=n$ as well. This completes STEP 2 and the induction argument. The existence of the sequence $\left\{\left(u_{k}, R_{k}, t_{k}, \bar{t}_{k}, \delta_{k}\right)\right\}$ with all the desired properties is now proved.

\section{References}

[1] P. Baras and L. Cohen, Complete blow-up after $T_{\text {max }}$ for the solution of a semilinear heat equation, J. Functional Anal. 71 (1987), 142-174.

[2] H. Berestycki and P.-L. Lions, Nonlinear scalar field equations. I. Existence of a ground state, Arch. Rational Mech. Anal. 82 (1983), 313-345.

[3] M.-F. Bidaut-Véron, Initial blow-up for the solutions of a semilinear parabolic equation with source term, Équations aux dérivées partielles 
et applications, Gauthier-Villars, Éd. Sci. Méd. Elsevier, Paris, 1998, pp. 189-198.

[4] T. Cazenave and P.-L. Lions, Solutions globales d'équations de la chaleur semi linéaires, Comm. Partial Differential Equations 9 (1984), 955-978.

[5] X. Chen, M. Fila, and J.-S. Guo, Boundedness of global solutions of a supercritical parabolic equation, Nonlinear Anal. 68 (2008), 621-628.

[6] M. Fila, Boundedness of global solutions of nonlinear diffusion equations, J. Differential Equations 98 (1992), 226-240.

[7] M. Fila and J. R. King, Grow up and slow decay in the critical Sobolev case, Netw. Heterog. Media 7, 661-671, (2012).

[8] M. Fila, J. R. King, M. Winkler, and E. Yanagida, Optimal lower bound of the grow-up rate for a supercritical parabolic equation, J. Differential Equations 228 (2006), 339-356.

[9] M. Fila, M. Winkler, and E. Yanagida, Grow-up rate of solutions for a supercritical semilinear diffusion equation, J. Differential Equations 205 (2004), 365-389.

[10] H. Fujita, On the blowing up of solutions of the Cauchy problem for $u_{t}=\Delta u+u^{1+\alpha}$, J. Fac. Sci. Univ. Tokyo Sect. I 13 (1966), 109-124.

[11] V. A. Galaktionov and J. L. Vázquez, Continuation of blow-up solutions of nonlinear heat equations in several space dimensions, Comm. Pure Applied Math. 50 (1997), 1-67.

[12] Y. Giga, A bound for global solutions of semilinear heat equations, Comm. Math. Phys. 103 (1986), 415-421.

[13] C. Gui, W.-M. Ni, and X. Wang, On the stability and instability of positive steady states of a semilinear heat equation in $\mathbb{R}^{n}$, Comm. Pure Appl. Math. 45 (1992), 1153-1181.

[14] A. Haraux and F. B. Weissler, Nonuniqueness for a semilinear initial value problem, Indiana Univ. Math. J. 31 (1982), 167-189.

[15] B. Hu, Blow-up theories for semilinear parabolic equations, Lecture Notes in Mathematics, vol. 2018, Springer, Heidelberg, 2011. 
[16] A. A. Lacey and D. Tzanetis, Complete blow-up for a semilinear diffusion equation with a sufficiently large initial condition, IMA J. Appl. Math. 41 (1988), 207-215.

[17] H. A. Levine, The role of critical exponents in blowup theorems, SIAM Rev. 32 (1990), 262-288.

[18] H. Matano and F. Merle, On nonexistence of type II blowup for a supercritical nonlinear heat equation, Comm. Pure Appl. Math. 57 (2004), 1494-1541.

[19]_, Classification of type I and type II behaviors for a supercritical nonlinear heat equation, J. Funct. Anal. 256 (2009), 992-1064.

[20] N. Mizoguchi, On the behavior of solutions for a semilinear parabolic equation with supercritical nonlinearity, Math. Z. 239 (2002), 215-229.

[21] _ Boundedness of global solutions for a supercritical semilinear heat equation and its application, Indiana Univ. Math. J. 54 (2005), 1047-1059.

[22] _ Growup of solutions for a semilinear heat equation with supercritical nonlinearity, J. Differential Equations 227 (2006), 652-669.

[23] P. Poláčik and P. Quittner, A Liouville-type theorem and the decay of radial solutions of a semilinear heat equation, Nonlinear Analysis, TMA 64 (2006), 1679-1689.

[24] P. Poláčik, P. Quittner, and Ph. Souplet, Singularity and decay estimates in superlinear problems via Liouville-type theorems. Part II: Parabolic equations, Indiana Univ. Math. J. 56 (2007), 879-908.

[25] P. Poláčik and E. Yanagida, On bounded and unbounded global solutions of a supercritical semilinear heat equation, Math. Ann. 327 (2003), 745771.

[26] P. Quittner, A priori bounds for global solutions of a semilinear parabolic problem, Acta Math. Univ. Comenian. (N.S.) 68 (1999), 195-203.

[27] P. Quittner and Ph. Souplet, Superlinear parabolic problems. blowup, global existence and steady states, Birkhäuser Advanced Texts, Birkhäuser, Basel, 2007. 
[28] X. Wang, On the Cauchy problem for reaction-diffusion equations, Trans. Amer. Math. Soc. 337 (1993), 549-590. 